\title{
Spatial Noise Source Identification of Tonal Noise in Turbomachinery Using the Coherence Function on a Modal Basis
}

\author{
Ian Davis* Gareth J. Bennett ${ }^{\dagger}$ \\ Trinity College Dublin, Ireland.
}

\begin{abstract}
In modern helicopter engines, the core noise generated by the combustor and turbine stages dominates the core sound radiated from the engine exhaust. Identification of the specific contributions of each rotor-stator stage to the core noise is impeded by the lack of available space and the complex duct geometry within the engine. Modal decomposition is an advanced technique which can be used to analyse detailed information on the modal content of the core noise radiating from the engine. This paper advances previous work whereby a technique of identifying noise source location in ducts using the coherence function on a modal basis was developed. A proof of concept was developed using analytical data, with a full radial modal decomposition performed at two axial locations upstream and downstream in the duct. For the case of a real helicopter engine however, there is not enough space to perform a full modal decomposition upstream of the turbine. In this paper, the aforementioned technique is applied to experimental data in order to verify its usefulness for application inside a real turboshaft engine. The technique is also advanced to calculate the coherence between the modes found from a full modal decomposition close to the exit plane of the test duct in the flow (analogous to the engine exhaust) and a single pressure measurement upstream (analogous to a point inside the engine close to a noise source of interest). This better represents a realisable technique within the small confines of an engine, as only one pressure transducer is required in the region of each source location of interest. Experimental data measured within an FP7 European research project by one of the consortium partners (German Aerospace Centre (DLR)) was be used to validate this technique.
\end{abstract}

\section{Introduction}

The study presented in this paper is a continuation of recent work undertaken by Bennett et al, ${ }^{1}$ where a novel method of identifying the location of a tonal noise source was developed and tested using analytically generated data. This data was produced by simulating the sound field generated by several point monopole sources in a hard walled annular flow duct. By applying a formulation by Goldstein ${ }^{2}$ of the frequencydomain Greens function for a duct with an axial irrotational mean flow, the sound pressure generated by each monopole can be simulated. Details of the technique tested and the test set-up are given in section III, and full details of the analytical test results can be found in the original paper. The aim of the work presented in this paper is to study the effectiveness of this technique for an experimental scenario which is very similar to the test scenario investigated for the analytical study.

\section{Modal Decomposition Technique}

Modal decomposition is an advanced technique whereby the specific amplitudes of the acoustic modes at a given axial location can be found. The modal decomposition technique applied herein uses an array of acoustic pressure sensors to decompose the pressure field into its constituent modal amplitudes, and allows the amplitude of any $(\mathrm{m}, \mathrm{n})$ mode cut-on in an allowable frequency range to be found. The technique also

*Ph.D. Student, Dept. of Mechanical and Manufacturing Engineering, Trinity College, Dublin 2, Ireland.

$\dagger$ Lecturer, Dept. of Mechanical and Manufacturing Engineering, Trinity College, Dublin 2, Ireland. 
allows the directionality of these modes to be found i.e. the amplitudes of the modes travelling in either axial direction can be determined. Modal decomposition techniques make use of the fact that assuming an incompressible and isentropic flow, stationary mean temperature and density and a constant axial mean flow profile, the Helmholtz equation (in cylindrical coordinates) can be expressed as the linear superposition of modal terms:

$$
p(x, r, \varphi)=\sum_{m=-\infty}^{\infty} \sum_{n=0}^{\infty}\left(A_{m n}^{+} e^{i k_{m n}^{+} x}+A_{m n}^{-} e^{i k_{m n}^{-} x}\right) f_{m n}(r) e^{i m \varphi}
$$

where $k_{m n}^{+}$and $k_{m n}^{-}$denote the axial wavenumbers, $A_{m n}^{+}$and $A_{m n}^{-}$denote the complex amplitudes for mode order $(\mathrm{m}, \mathrm{n})$, where $m$ is the azimuthal mode order and $n$ is the radial mode order. $f_{m n}(r)$ is the modal shape factor for mode order $(\mathrm{m}, \mathrm{n})$, which is formulated by a combination of Bessel functions and is dependant on the cross-sectional geometry of the duct.

Many modal decomposition techniques have been proposed and applied in the literature. Enghart,, 4 Tapken, ${ }^{5,6}$ Karchmer, ${ }^{7}$ Holste and Neise ${ }^{8}$ and Bennett ${ }^{9}$ for example have applied such techniques to decompose the pressure field at a given axial location into its specific modal amplitudes. The modal decomposition technique used in this paper is based on Åbom's ${ }^{10}$ technique, as described in chapter 7 of Bennett. ${ }^{9}$ This technique is carried out in two stages. Firstly, an azimuthal decomposition is carried out with microphones equi-azimuthally spaced around the duct:

$$
p_{l, k}=\sum_{m=1-M}^{M-1} h_{m, k} e^{\left[j m \theta_{l}\right]}
$$

By repeating this stage at different axial locations, these modes can be decomposed into incident and reflected radial modes. This second stage uses the following equation:

$$
h_{m k}=\sum_{n=0}^{N-1}\left[A_{m, n}^{+} e^{-j k_{m, n}^{+} x}+A_{m, n}^{-} e^{+j k_{m, n}^{-} x}\right] f_{m, n}(r)
$$

From this equation the modal amplitudes $A_{m, n}^{ \pm}$can be determined. This method requires that at least two measurements are taken per azimuthal wavelength $\left(\frac{2 \pi}{m}\right)$, so that each azimuthal mode can be uniquely determined (see Holste and Neise ${ }^{8}$ and $\AA$ bom $^{10}$ for similar reasoning).

\section{Spatial Coherence of Acoustic Modes}

The coherence function $\gamma_{i j}^{2}(f)$ of two time-domain signals $i(t)$ and $j(t)$ is found from the cross-spectral density and auto-spectral density functions of the two functions. These spectral density functions are averaged over many signal blocks. An example of such time-domain measurements are microphone measurements, which measure the acoustic pressure with time. For all frequencies $(f)$ the quantity $\gamma_{i j}^{2}(f)$ satisfies $0 \leq \gamma_{i j}^{2}(f) \leq 1$. The situation where the coherence function may be less than one but greater than unity may be explained by the following physical situations:

- Extraneous noise is present in the measurements.

- The system relating $i(t)$ and $j(t)$ is not linear.

- $j(t)$ is an output due to input $i(t)$ as well as other inputs.

If $i(t)$ and $j(t)$ are simply unrelated, the coherence will be zero. The technique implemented in this paper applies the coherence function on a modal basis, where the modal amplitudes have been determined at two different axial locations. The coherence function is used as a tool in this paper on the assumption that if a specific mode order has a significant amplitude at two different axial locations, the coherence at this mode order will be high. As the complex modal amplitudes (with directionality) can be found using modal decomposition, auto- and cross-spectra can be formulated and hence the coherence function can be calculated on a modal basis. This technique has been tested with numerical data by Bennett et al ${ }^{1}$ for the test scenario of two mode generation locations and two modal decomposition locations. The scenario being examined in this paper is similar to this, with two locations of mode generation and two locations of 
modal decomposition. The locations of mode generation are the rotor-stator stage and a mode generator respectively. The coherence function can be expressed on a modal basis by:

$$
\gamma_{A_{m, n}^{ \pm}(X), A_{m, n}^{ \pm}(Y)}^{2}=\frac{\left|G_{A_{m, n}^{ \pm}(X), A_{m, n}^{ \pm}(Y)}\right|^{2}}{G_{A_{m, n}^{ \pm}(X), A_{m, n}^{ \pm}(X)} G_{A_{m, n}^{ \pm}(Y), A_{m, n}^{ \pm}(Y)}}
$$

This equation allows the coherence to be found between the modes measured at two different axial locations. As the modal decomposition technique shown in section II can be used to determine the amplitudes of the modes at an axial location with directionality, the coherence can be calculated between modes travelling in either direction at two sensor banks. The schematic for the test scenario investigated in this study is shown below in Figure 1. It is a two source region model. These noise source regions are a rotor-stator stage and a mode generator. A mode generator is simply an array of loudspeakers generating tonal noise. By adjusting the phase relationship between these individual loudspeaker sources, a specific azimuthal mode can be targeted by the mode generator array as per Equation 7. The rotor-stator will generate a broadband noise floor, with tonal peaks at the BPF (blade-pass frequency) and its harmonics. The dominant azimuthal mode generated at the BPF by a rotor-stator stage with $B$ rotor blades and $V$ stator vanes is determined by Tyler-Sofrin theory, ${ }^{11}$ given in Equation 5. The upstream location of modal decomposition is denoted location $\mathbf{X}$ and the downstream location of modal decomposition is denoted location $\mathbf{Y}$.

$$
\begin{gathered}
m_{\text {rot }}=n B-k V \\
q\left(\theta_{l}\right)=q_{m} e^{\left(i m \frac{2 \pi l}{S}\right)} \quad \text { where } \begin{array}{l}
l=0,1, \ldots,(S-1) \\
\theta_{l}=\frac{2 \pi l}{S}
\end{array}
\end{gathered}
$$

One key factor in the experimental tests is the presence of reflections not present in previous analytical tests. Sound will be reflected at the downstream open end and at the rotor-stator stage. The red modes shown in Figure 1 are anticipated modal reflections resulting from this. The added value of the modal coherence technique is the ability to identify the location of a given noise source by investigating the coherence between the modes at two spatial locations travelling in either axial direction. For example, if the coherence between $A_{m, n}^{-}(X)$ and $A_{m, n}^{-}(Y)$ is high for a given $(\mathrm{m}, \mathrm{n})$ mode order at a certain frequency $(f)$, it can be concluded that a noise source exists downstream of $\mathbf{X}$ and $\mathbf{Y}$ which is generating significant acoustic energy at that mode at frequency $f$. If however the reflections are high at both ends of the test duct shown in Fig. 1, the technique will fail to provide any useful information on the location of either source, as the coherences will be high for all modes present travelling in both axial directions. It is therefore necessary that at least one end of the test duct terminates anechoically, eliminating or severely decreasing the amplitudes of any reflections.

As well as testing the original technique where the pressure field is decomposed into its modal constituent amplitudes at two axial locations, an alternative technique is also presented. In this technique, the modal amplitudes are found at one axial location, and the coherence function is formulated between these complex modal amplitudes and a single pressure measurement at another axial location in the duct. This technique reduces the logistical issues of the spatial coherence technique, as only one reference sensor is required close to the source region of interest. In a real turboshaft engine, this is a more realistic scenario, as the complex duct geometry and lack of available space for the location of an array of sensors means that a modal decomposition may not be possible inside the engine. With reference to Figure 1, the coherence function could be calculated between the complex amplitudes of the modes travelling in the negative z-direction at location $\mathbf{X}$ and a single acoustic pressure sensor located at one of the noise source regions present in the test duct. By examining the coherences for each mode order with frequency, the contribution of this noise source region to the modal content at location $\mathbf{X}$ can be identified. For example, by locating a reference sensor close to the mode generator, the contribution of the mode generator array to the modal content travelling towards the duct inlet plane can be identified. This technique requires a pure measurement of the source 


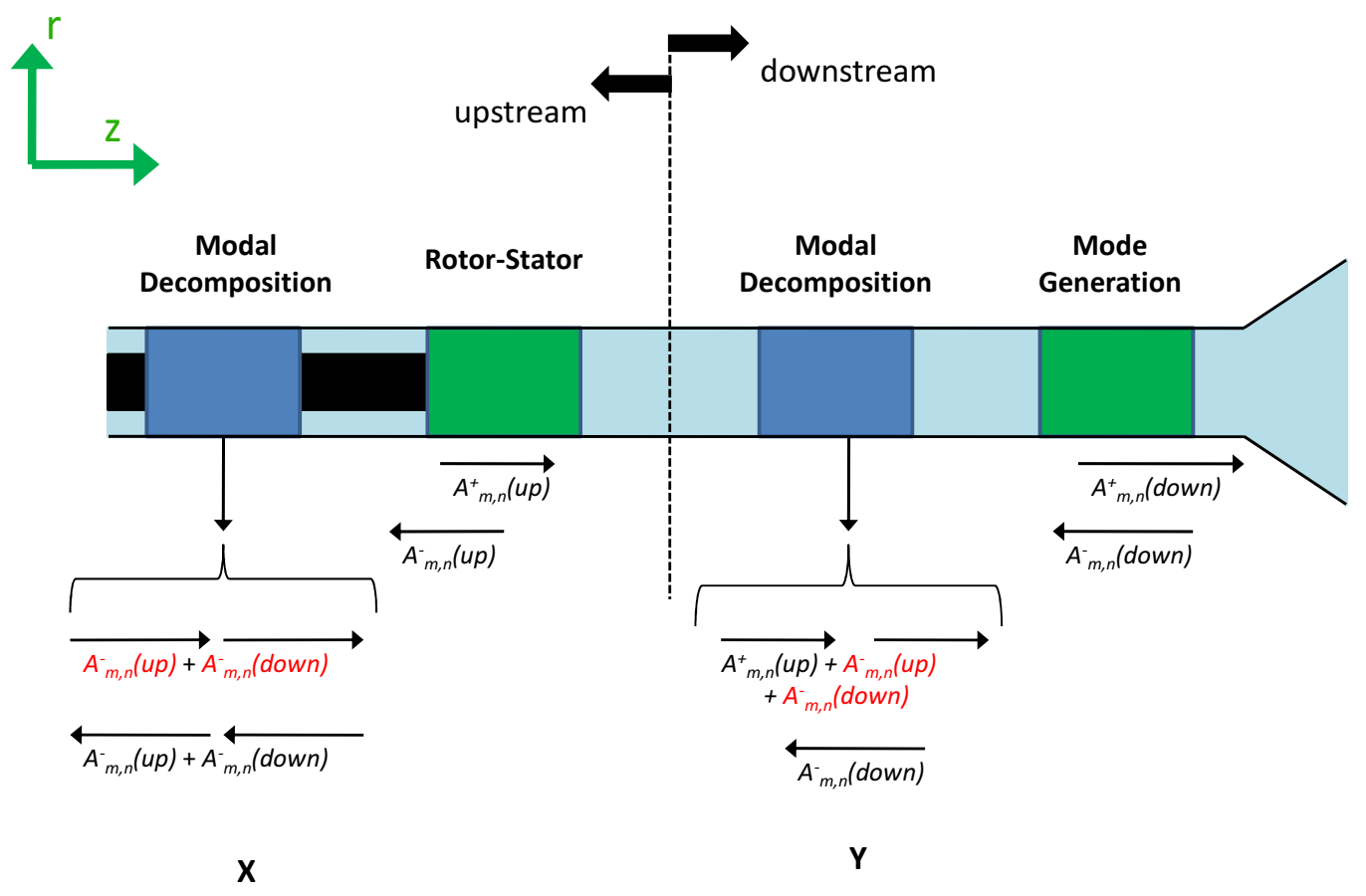

Figure 1: Schematic of modal coherence scenario investigated using experimental data (modal reflections in red). The rotating rotor will induce flow in the positive z-direction. 
region of interest, i.e. the reference sensor must measure the source of interest and must not measure any other noise sources which may be present. Furthermore, the presence of any additional extraneous noise such as flow noise at the reference sensor will contaminate the result.

\section{Experimental Rig}

The experimental rig shown in Figure 2 was used to test the techniques proposed in this paper. This experimental set-up was designed by the DLR Engine Acoustics group to simulate the main acoustic elements downstream of the combustor in a turboshaft engine. This paper focuses on one specific test point of the test campaign. At this test point, the sources of noise in the rig are a single rotor-stator stage consisting of 24 rotor blades rotating at $3000 \mathrm{rpm}$ and 5 stationary stator vanes, and an array of 16 loudspeakers in a single axial ring generating tonal noise. This array of loudspeakers will be referred to as the mode generator. The duct terminates anechoically at the downstream end.

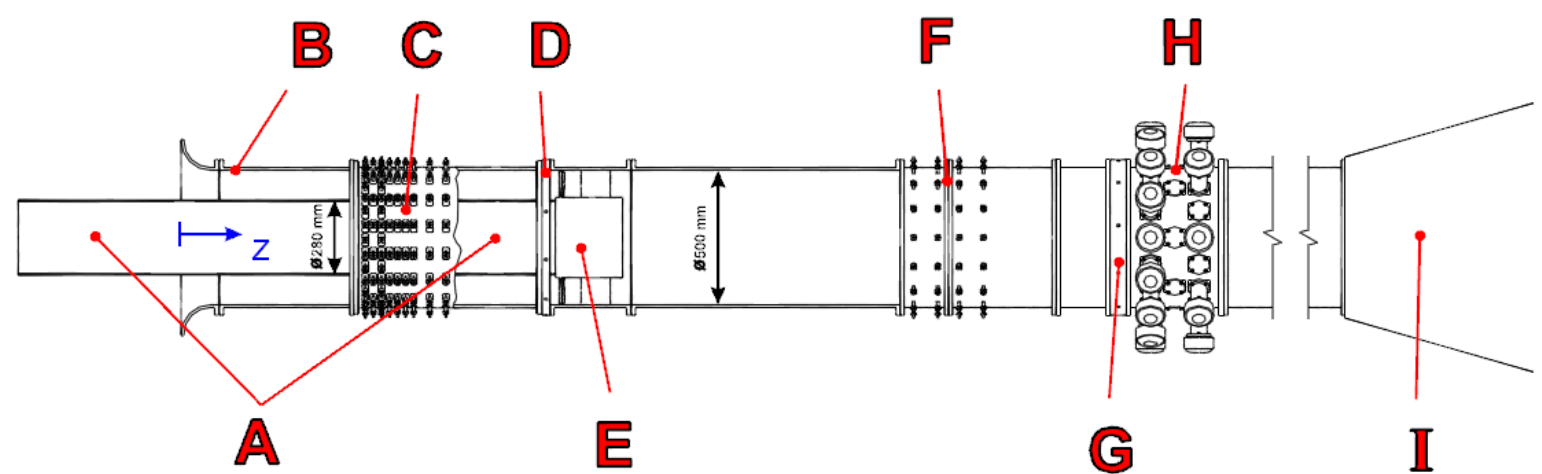
A: hub
$B$ : inlet duct
C: inlet array
D: ring with reference sensors
$\mathrm{E}$ : rotor-stator stage
$F$ : outlet array
G: ring with reference sensors
$\mathrm{H}$ : mode generator
I: anechoic termination

Figure 2: Schematic of DLR Rig.

Data was acquired at 256 independent ADC (analogue-to-digital) channels using in-house hardware and software at a sampling frequency of $16 \mathrm{kHz}$. G.R.A.S. Sound and Vibration condenser type 40BP microphones were used to acquire the acoustic pressures. Two banks of 64 receivers were located in the test duct, one of which was located at the hub of the rig. The hub-to-tip ratio at this location, $\eta$, is 0.56 . These sensor arrays were used to determine the modal amplitudes as per the technique outlined in section II. Eleven reference sensors were also located in the duct close to both the mode generator and rotor-stator source regions.

The rotor-stator generates a BPF tone at $1210 \mathrm{~Hz}$ at $3000 \mathrm{rpm}$, with the $m=-1$ azimuthal mode dominating as predicted using Equation 5. The rotating rotor induces a mean flow in the positive z-direction of $17.76 \mathrm{~m} / \mathrm{s}$ at the duct inlet. The mode generator consists of 16 loudspeakers in a single axial ring targeting the zeroth azimuthal mode at a tonal frequency of $800 \mathrm{~Hz}$ using the phase relationship between the speakers, as shown in Equation 7.

\section{Results}

The amplitudes of the modes travelling in both z-directions at both sensor banks are shown in Fig. 3. These sensor banks are denoted $\mathbf{X}$ and $\mathbf{Y}$. The modal amplitudes are shown for all azimuthal mode orders 
which are cut-on at both sensor banks at both 800 and $1210 \mathrm{~Hz}$. At the frequency of $800 \mathrm{~Hz}$, the mode generator is the main contributor to the acoustic energy propagating in the negative z-direction. The zeroth azimuthal mode dominates, as this is the mode targeted by the mode generator. Due to reflections at the rotor stage and at the inlet of the duct, the mode generator also contributes to the noise travelling in the positive z-direction at this frequency, and the zeroth mode again dominates. At $1210 \mathrm{~Hz}$, which corresponds to the rotor BPF, the modal content at locations $\mathbf{X}$ and $\mathbf{Y}$ differs considerably. This is due to the fact that the stator is located in the negative z-direction relative to the rotor. The rotor-stator interaction tone, with the $m=-1$ mode dominating, is therefore clearly evident in the amplitudes of the modes travelling in the negative z-direction at location $\mathbf{X}$ at $1210 \mathrm{~Hz}$. However, at location $\mathbf{Y}$, the dominance of the $m=-1$ mode is not evident. This is caused by the fact that the interaction tone propagates in the negative z-direction from the rotor, is reflected at the inlet, and the reflected component propagates in the positive z-direction and is measured at $\mathbf{Y}$. The modal amplitudes at location $\mathbf{X}$ demonstrate that the sound is weakly reflected at the inlet, as shown by the significant difference in amplitude between the modes travelling in both directions. Other rotor noise besides rotor-stator interaction noise will also propagate in the positive z-direction and contribute to the modal energy at location $\mathbf{Y}$. The amplitudes of the modes travelling in the negative $\mathrm{z}-$ direction at location $\mathbf{Y}$ at the rotor BPF are considerably lower than the amplitudes of the modes travelling in the positive z-direction - this is due to the presence of an anechoic termination at the downstream end of the duct, which should considerably reduce the amplitudes of any reflections at this end of the duct.
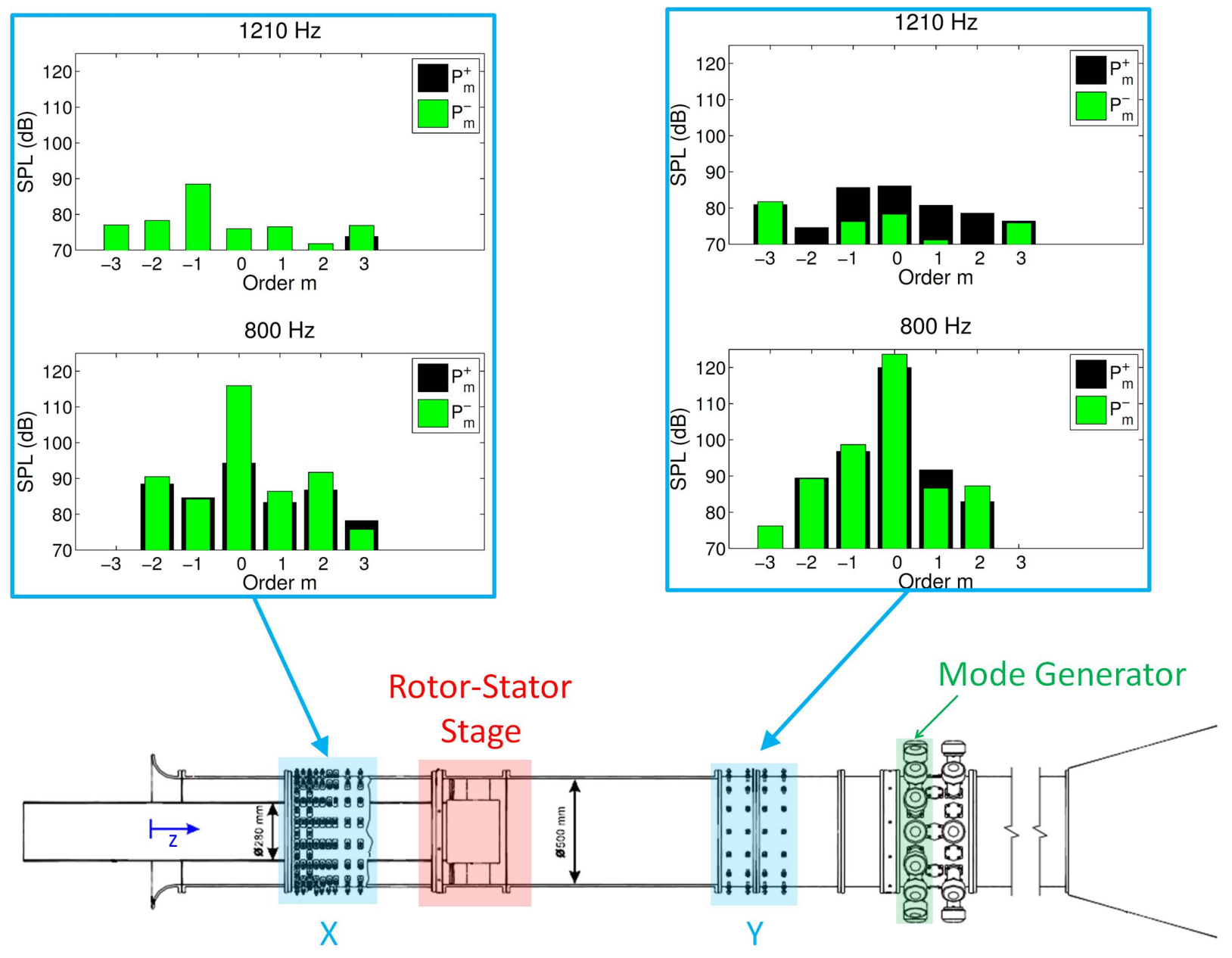

Figure 3: Schematic showing noise source and modal decomposition locations in the test duct. Two noise source regions are present - a rotor rotating at $3000 \mathrm{rpm}$, generating a $\mathrm{BPF}$ tone at $1210 \mathrm{~Hz}$, and a mode generator generating a tone at $800 \mathrm{~Hz}$. The amplitudes of the modes travelling in the positive and negative z-directions are decomposed at sensor banks $\mathrm{X}$ and $\mathrm{Y}$. These modal amplitudes are shown at the mode generator tonal frequency and the rotor BPF. 
The presence of an anechoic termination at the downstream end should reduce, if not eliminate, the presence of reflections at location $\mathbf{Y}$ in the negative z-direction. This would be demonstrated experimentally by a low reflection of the rotor BPF which would propagate in the negative z-direction. Figure 3 demonstrates that although reflections are minimised, they are not eliminated. This would only be possible if some energy at the BPF is being reflected at the downstream end of the duct. Since the BPF is of very high magnitude, around $30 \mathrm{~dB}$ above the noise floor (see Fig. 4), even a very low reflection coefficient at the downstream end will result in a significant tonal peak reflected at the downstream end and propagating in the negative z-direction. Figure 5 shows that the coherence between the modes propagating in the negative z-direction at locations $\mathbf{X}$ and $\mathbf{Y}$ is high at the BPF $(1210 \mathrm{~Hz})$.

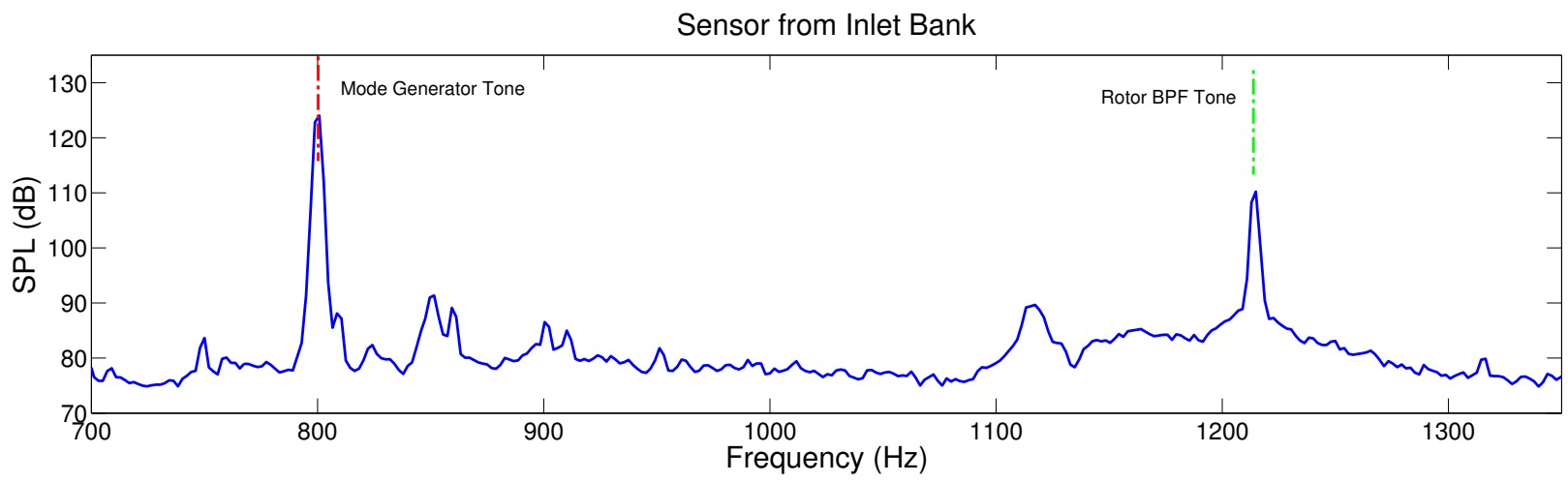

Figure 4: Spectrum of the sound pressure level at a sensor from the inlet sensor bank (see Fig. 2). The tones generated by both the mode generator and the rotor-stator are shown.

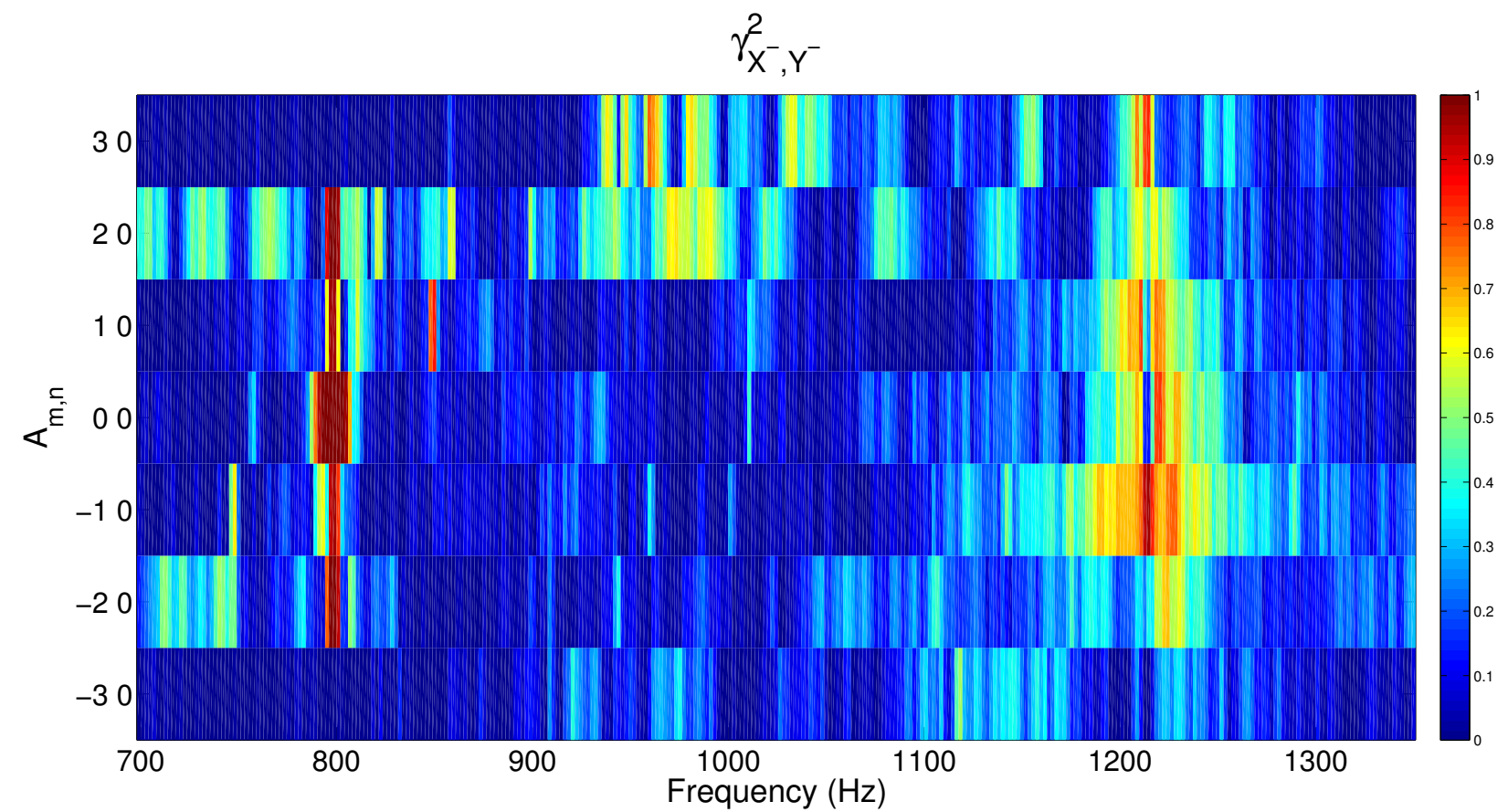

Figure 5: Checkerboard plot showing the coherence between modes travelling in the -z direction at upstream (X) and downstream (Y) banks of receivers. The colour of each rectangular box shows the coherence from 0 to 1 for a given acoustic mode at a specific frequency.

To eliminate this reflection, it was proposed that additional uncorrelated Gaussian noise be added at the post-processing stage. As in the previous paper documenting the analytical tests ${ }^{1}$ this noise was added to the time-domain acoustic pressure data measured by the microphones in both sensor arrays. Different 
levels of noise were added (see Table 1). These noise levels are a function of the standard deviation of the raw-spectra for each microphone measurement at both banks of receivers. These noise floor levels are shown relative to the raw spectra in Fig. 6. This represents a realistic scenario for a real turboshaft engine, as the broadband noise floor inside a real engine will be far higher than in these small-scale tests due to the presence of many core noise sources, such as at the combustor.

Table 1: Levels of noise added to time-domain pressure fields relative to the acoustic pressure signals measured by each sensor.

\begin{tabular}{|c|c|}
\hline Name & Noise Level \\
\hline Low & $0.2 \times$ Standard Deviation \\
Medium & $1 \times$ Standard Deviation \\
High & $4 \times$ Standard Deviation \\
\hline
\end{tabular}

Sensor from Downstream Sensor Bank
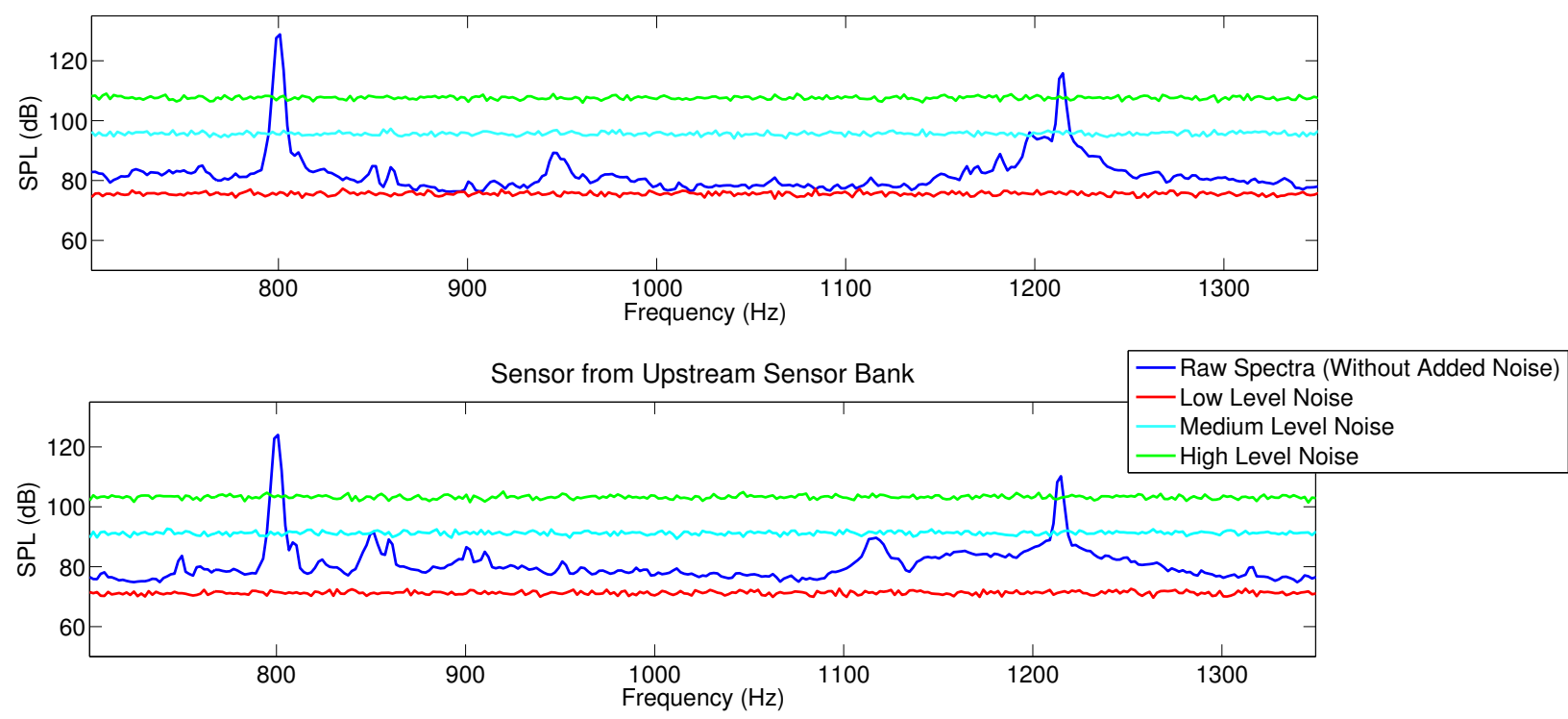

Figure 6: Auto-spectra of a microphone measurements from both the upstream and downstream banks of receivers (locations $X$ and $Y$ respectively). This figure shows the raw spectra and the different levels of noise added during post-processing.

Figure 7 shows how, when a high level of noise has been added, the coherence between the $(-1,0)$ modes travelling in the negative z-direction at the BPF of $1210 \mathrm{~Hz}$ at both banks $\mathbf{X}$ and $\mathbf{Y}$ drops to low background levels (see Figure 1). This shows that reflections at the upstream anechoic end are eliminated by adding uncorrelated noise to the time-domain pressure signals for each sensor at both banks of microphones. Figure 8 shows that, with the same magnitude of noise added, the peak in coherence of the $(-1,0)$ mode at the $\mathrm{BPF}$ propagating in the negative z-direction at the upstream $(\mathbf{X})$ and in the positive z-direction at the downstream $(\mathbf{Y})$ banks of receivers remains. The added noise has therefore not masked the incident BPF tone in the results.

The coherences between all modes cut-on in the frequency range of interest at both banks $\mathbf{X}$ and $\mathbf{Y}$ for the modes travelling in the negative z-direction, with high noise added, are shown in Figure 9. This checkerboard plot shows the high coherence for the zeroth mode at $800 \mathrm{~Hz}$, and low coherence at all other frequencies for all modes. It could thus be deduced correctly using this modal coherence techniques that a source region exists downstream of both banks $\mathbf{X}$ and $\mathbf{Y}$, which generates a tone at $800 \mathrm{~Hz}$, and the $(0,0)$ mode is dominant.

Similarly, Figure 10 shows the coherences between the modes propagating in the negative z-direction at location $\mathbf{X}$ and in the positive z-direction at location $\mathbf{Y}$. The coherence is again high at the mode generator 


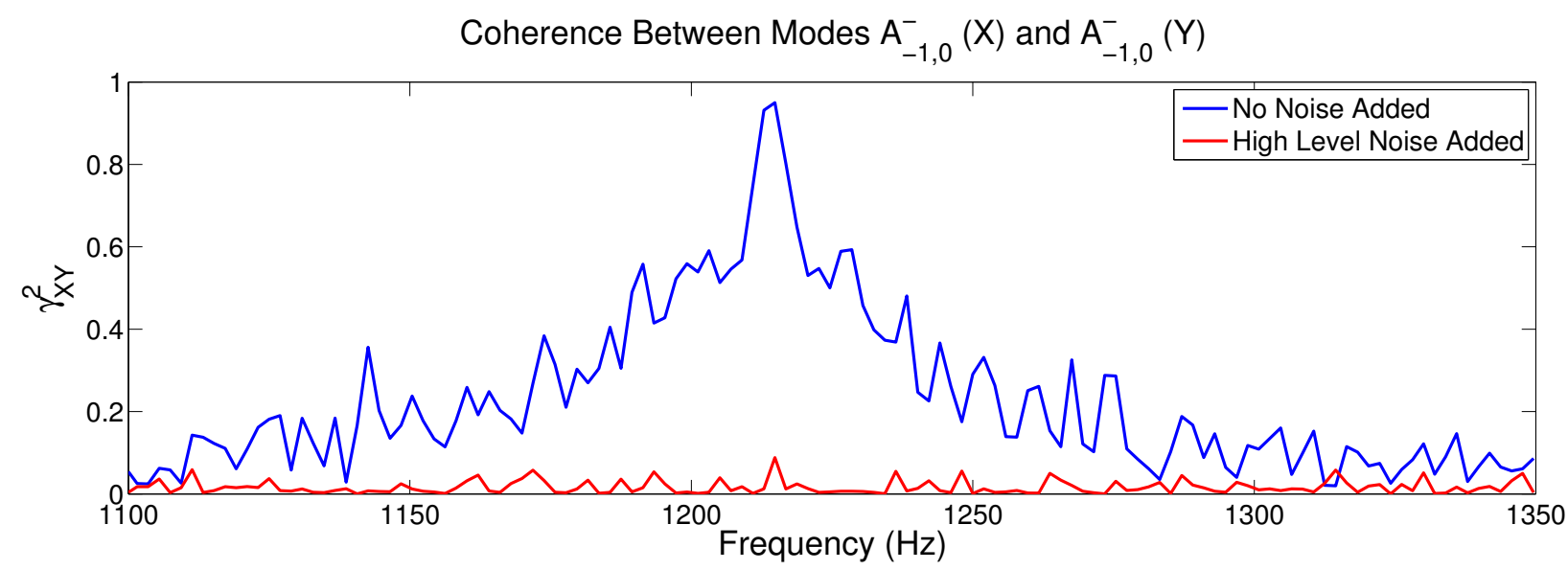

Figure 7: Coherence between $(-1,0)$ modes propagating in the negative z-direction at upstream (X) and downstream (Y) banks of receivers, showing the effect of added noise on the modal coherences.

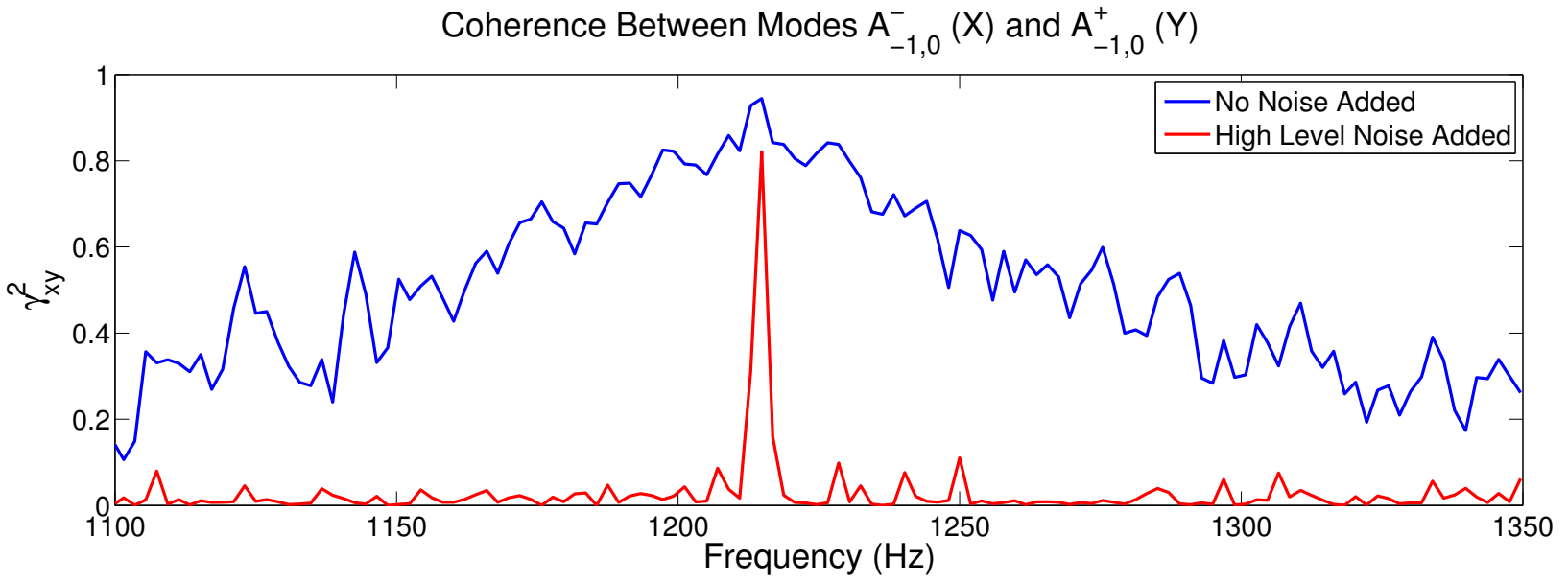

Figure 8: Coherence between $(-1,0)$ modes propagating in the negative z-direction at the upstream $(\mathrm{X})$ and in the positive z-direction at the downstream (Y) banks of receivers, showing the effect of added noise on the modal coherences. 
frequency of $800 \mathrm{~Hz}$. The coherence is also high at the rotor BPF of $1210 \mathrm{~Hz}$ for the $(-1,0)$ mode. As there can be no noise sources upstream of location $\mathbf{X}$, it can be concluded that a noise source region exits between locations $\mathbf{X}$ and $\mathbf{Y}$ which generates a tone at $1210 \mathrm{~Hz}$, with the $(-1,0)$ mode dominating.

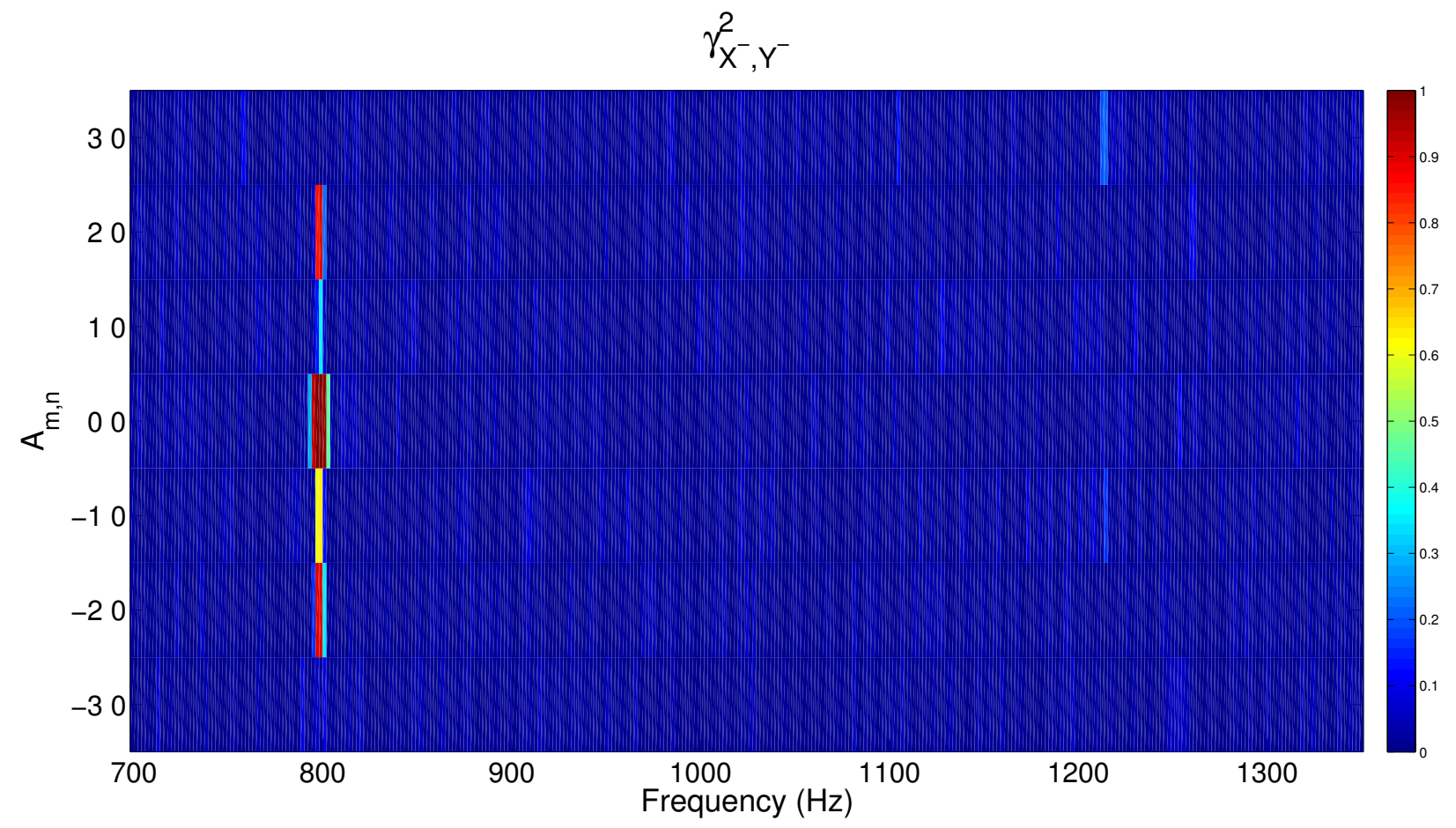

Figure 9: Checkerboard plot showing the coherence between modes travelling in the negative zdirection at upstream $(\mathrm{X})$ and downstream $(\mathrm{Y})$ banks of receivers, when high noise has been added. The colour of each rectangular box shows the coherence from 0 to 1 for a given acoustic mode at a specific frequency.

Figure 11 plots the coherences between the complex amplitudes of the modes travelling in the negative z-direction at location $\mathbf{X}$ and the complex pressure signal of a sensor located at the ring of reference sensors shown in $(G)$ of Figure 2. This ring of reference sensors is located close to the mode generator. The aim of the technique in this case is to successfully identify the contribution of the mode generator to this modal content, using only one reference sensor close to the source region. No additional noise was added to this sensor measurement. The technique works very well in this case, with the modal content generated by the mode generator clearly identified in the checkerboard plot at $800 \mathrm{~Hz}$. The BPF tone at $1210 \mathrm{~Hz}$ is not evident in the plot. This demonstrates that the reference sensor used does not measure the BPF at $1210 \mathrm{~Hz}$ above the added noise floor, and so the coherence is low at this frequency. The reference sensor therefore meets the requirement of the technique that the measurement of the source of interest (the mode generator) is pure i.e. only the source of interest is measured.

Figure 12 plots the coherence between the complex amplitudes of the modes travelling in the negative z-direction at location $\mathbf{X}$ and the complex pressure signal of a sensor located at the ring of reference sensors shown in (D) of Figure 2. This ring of reference sensors is located close to the rotor-stator stage. The aim of the technique in this case is to identify the contribution of the rotor-stator stage to the modal content travelling in the negative z-direction at the inlet sensor array. Although the coherence is high for the $(-1,0)$ mode at the BPF, which is a successful result, the high coherence at the mode generator frequency of 800 $\mathrm{Hz}$ across all cut-on modes is not. This result therefore does provide any information on its own, as it would suggest that the rotor-stator contributes significant modal energy at both the rotor BPF and mode generator frequencies. However, in a real turboshaft engine, if such a result was used in conjunction with prior knowledge of the rotor blade-pass frequency (through knowledge of the number of rotor blades and its rotational speed), the technique will provide information on the modal content which has propagated from the rotor-stator stage to the location of modal decomposition, such as the exit plane of the engine, at the rotor BPF. 


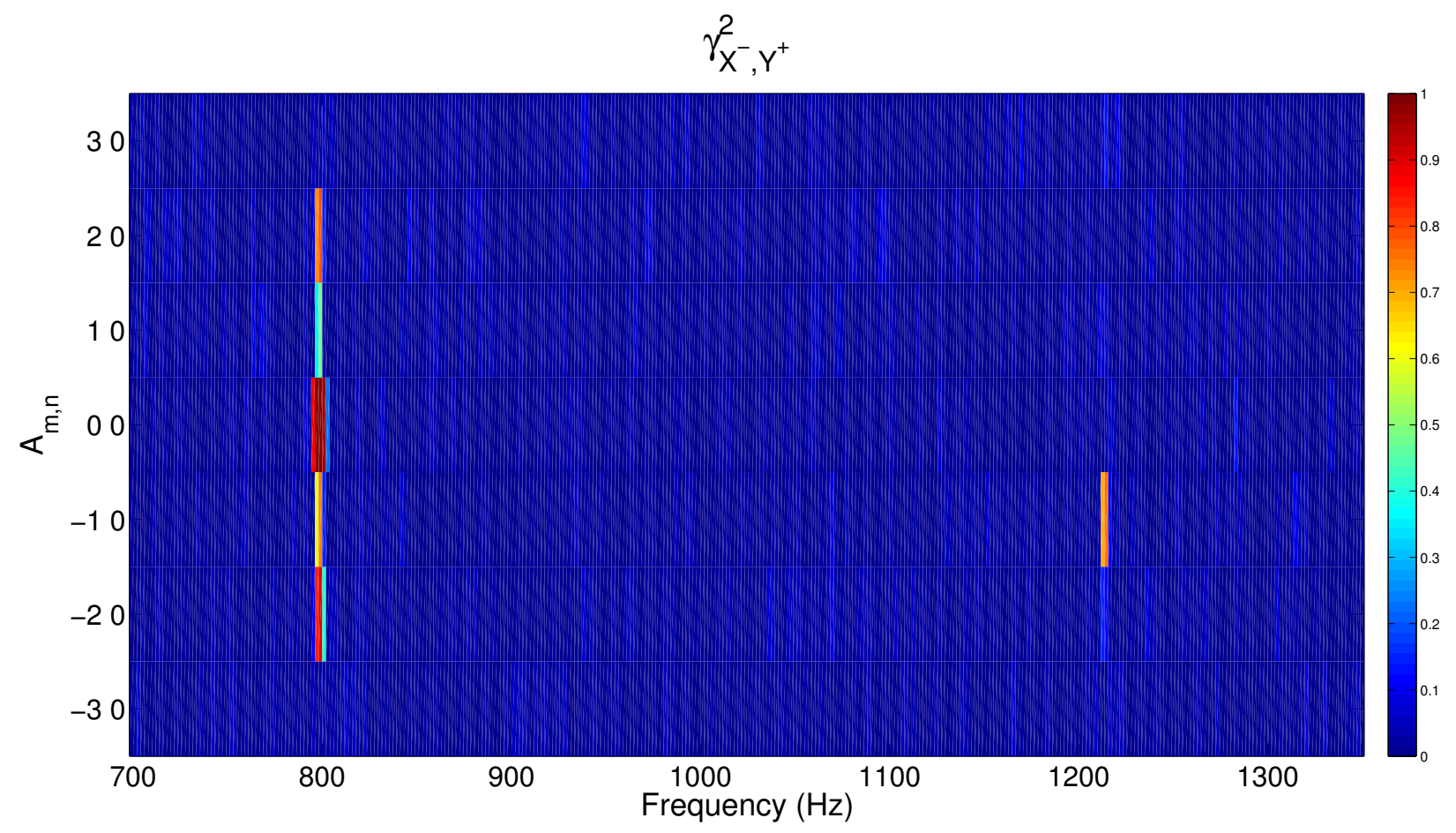

Figure 10: Checkerboard plot showing the coherence between the modes travelling in the negative z-direction at the upstream (X) bank and in the positive z-direction at the downstream (Y) banks of receivers, when a high level of noise has been added. The colour of each rectangular box shows the coherence from 0 to 1 for a given acoustic mode at a specific frequency.

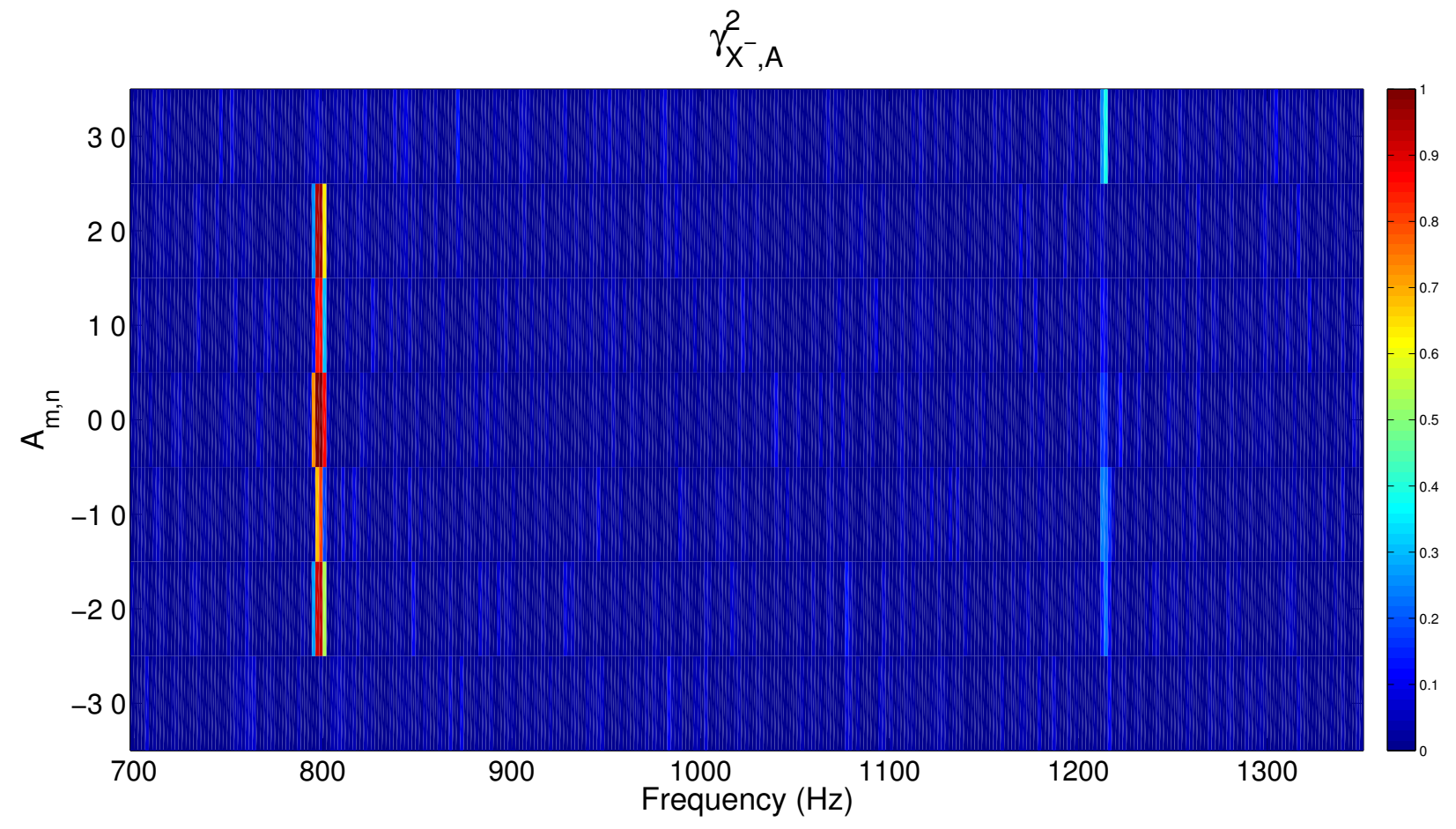

Figure 11: Checkerboard plot showing the coherence between modes travelling in the negative $\mathrm{z}-$ direction at the upstream (X) banks of receivers and a single sensor located close to the mode generator, when a high level noise of has been added. The colour of each rectangular box shows the coherence from 0 to 1 for a given acoustic mode at a specific frequency. 


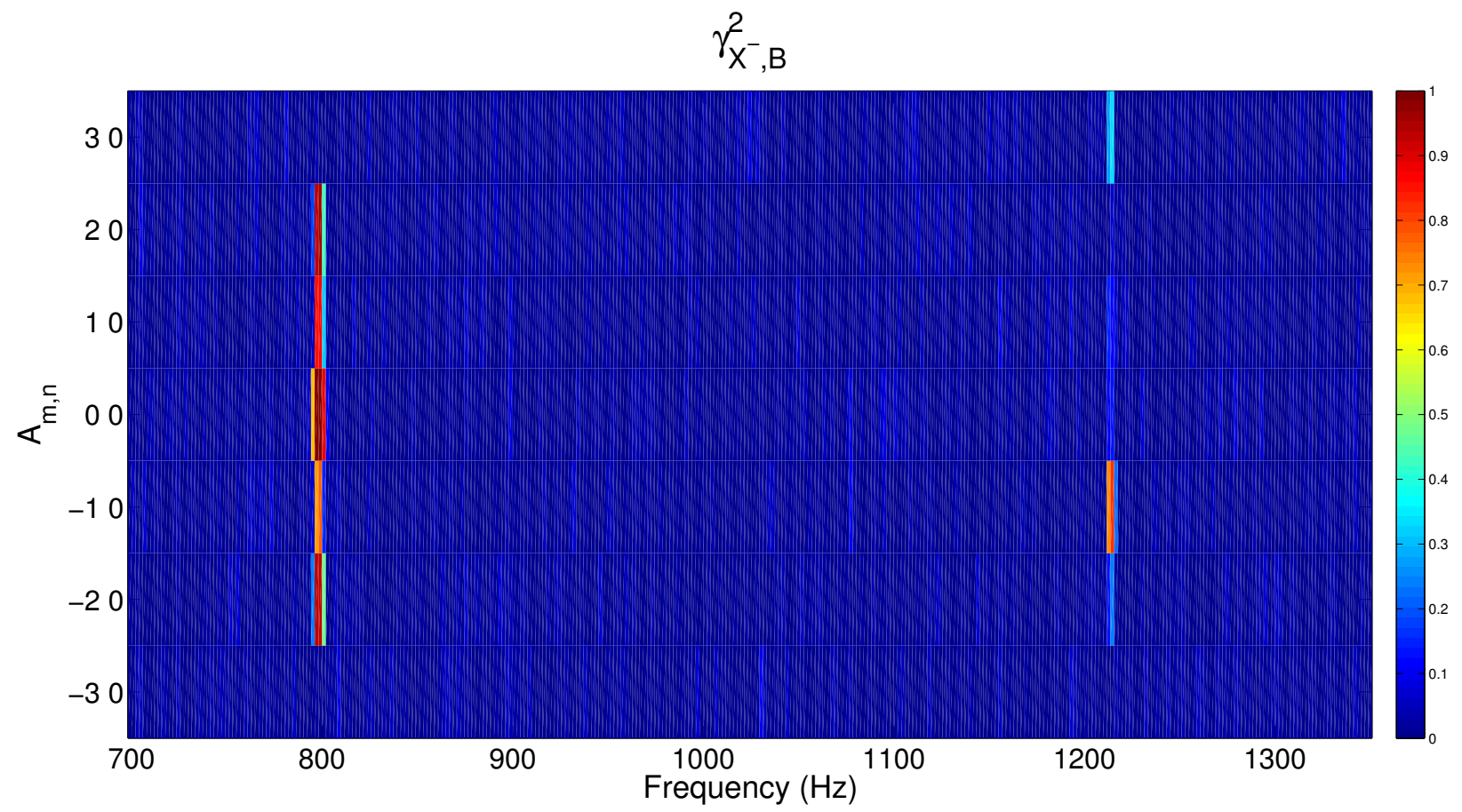

Figure 12: Checkerboard plot showing the coherence between modes travelling in the negative $\mathrm{z}-$ direction at the upstream (X) banks of receivers and a single sensor located close to the rotor-stator stage, when a high level of noise has been added. The colour of each rectangular box shows the coherence from 0 to 1 for a given acoustic mode at a specific frequency.

\section{Conclusions}

A technique for identifying the spatial location of a noise source using the coherence function on a modal basis has been investigated using experimental data, as generated in the TEENI small-scale rig test campaign. This technique had previously only been tested using analytically generated data. This paper demonstrates the benefits and limitations of the technique. As well as using the coherence function between the modes decomposed at two axial locations in the test duct, the coherence function was applied between the modes decomposed and a single acoustic pressure sensor located close to a noise source of interest in the test duct. This technique can be used in conjunction with prior knowledge of the noise generation mechanism of the noise source of interest to identify the contribution of the noise source to this modal content. This technique will be further experimentally investigated for a full-scale turboshaft engine in future tests.

\section{Acknowledgements}

This work was supported by the Seventh Framework Programme TEENI project which is funded under EU commission grant agreement 212367. The contribution of Mr Ulf Tapken, Mr Benjamin Pardowitz and Mr Philip Kausche to the experimental testing is gratefully acknowledged.

\section{References}

${ }^{1}$ G. Bennett, C. O'Reilly, U. Tapken, and J.A. Fitzpatrick. Noise Source Location in Turbomachinery Using Coherence Based Modal Decomposition. In 15th AIAA/CEAS Aeroacoustics Conference, Miami, Florida, 11-13 May. American Institute of Aeronautics and Astronautics, 2009.

${ }^{2}$ M.E. Goldstein. Aeroacoustics. McGraw-Hill International Book Company, 1976.

${ }^{3}$ L. Enghardt, Y. Zhang, and W. Neise. Experimental verification of a radial mode analysis technique using wall-flush mounted sensors. The Journal of the Acoustical Society of America, 105:1186, 1999.

${ }^{4}$ L. Enghardt, U. Tapken, W. Neise, F. Kennepohl, and K. Heinig. Turbine blade/vane interaction noise - Acoustic mode analysis using in-duct sensor rakes. In 7th AIAA/CEAS Aeroacoustics Conference, Maastricht, Netherlands, May 28-30, pages 
275-282, 2001.

${ }^{5}$ U. Tapken, L. Enghardt, W. Neise, P. Schimming, and L. Turbulenzforschung. Active Control of Noise from Turbomachines - Results of Radial Mode Analysis. In 30th International Congress on Noise Control Engineering (Internoise 2001), Den Haag, Netherlands, 2001.

${ }^{6} \mathrm{U}$. Tapken and L. Enghardt. Optimisation of sensor arrays for radial mode analysis in flow ducts. In 12th AIAA/CEASAeroacoustics Conference, Cambridge, MA, pages 8-10, 2006.

${ }^{7}$ A. Karchmer. Acoustic modal analysis of a full-scale annular combustor. In 8th AIAA Aeroacoustics Conference, Atlanta, GA, 1983.

${ }^{8}$ F. Holste and W. Neise. Noise source identification in a propfan model by means of acoustical near field measurements. Journal of Sound and Vibration, 203(4):641-665, 1997.

${ }^{9}$ G. Bennett. Noise Source Identification For Ducted Fans. PhD thesis, Trinity College Dublin, 2006.

${ }^{10} \mathrm{M}$. Åbom. Modal decomposition in ducts based on transfer function measurements between microphone pairs. Journal of Sound Vibration, 135:95-114, 1989.

${ }^{11}$ J.M. Tyler and T.G. Sofrin. Axial compressor noise studies. SAE Transactions, 70:309-332, 1962. 